\title{
MULHERES MASTECTOMIZADAS EM VIGÊNCIA DE QUIMIOTERAPIA ADJUVANTE: ASSISTÊNCIA DO ENFERMEIRO.
}

\author{
Débora Tavares Fernandes ${ }^{1 *}$, Marcella Vieira Manhães ${ }^{2}$, Virgínia Correa de O.B. Sousa ${ }^{2}$ \& \\ Carolina Magalhães dos Santos ${ }^{2}$
}

FERNANDES, D.T.; MANHÃES, M.V.; SOUSA, V.C.O.B.; \& SANTOS, C.M. Mulheres mastectomizadas em vigência de quimioterapia adjuvante: assistência do enfermeiro. Perspectivas Online: Biológicas \& Saúde. v. 8 , n 26 , p.19-30, 2018.

\section{RESUMO}

O presente estudo visa conhecer a forma como o profissional enfermeiro atua nas orientações acerca dos efeitos colaterais em pacientes mastectomizadas que se encontram em tratamento quimioterápico adjuvante. A pesquisa foi realizada na UNACON (Unidade Assistência de Alta Complexidade em Oncologia) do Instituto de Medicina Nuclear e Endocrinologia (IMNE/ONCOBEDA). A amostra da pesquisa foi formada por 30 mulheres mastectomizadas entre 30 e 45 anos. O processo de pesquisa foi iniciado através da leitura dos prontuários, sendo realizadas visitas ao referido Hospital para a coleta dos dados. Esta foi utilizada para formulação de um questionário, posteriormente aplicado às mulheres. Encontrou-se uma assistência de qualidade, e satisfação por parte das clientes nas orientações prestadas pelo enfermeiro, tal como os efeitos colaterais mais frequentes (alopecia, vômito, náuseas, afta, perda de apetite, diarreia, depressão, acesso venoso periférico comprometido (AVPC) e anemia) e os que mais causaram incomodo. Podemos concluir que o Enfermeiro possui um papel primordial nas orientações prestadas às clientes, esclarecendo as dúvidas, facilitando a sua aceitação e o seu enfrentamento, suprindo assim, as necessidades de cuidado apresentadas durante $\mathrm{o}$ tratamento.

Palavras-chave: Enfermagem; Mastectomia; Quimioterapia Adjuvante; Assistência do Enfermeiro. 


\begin{abstract}
The present study aims to know how the professional nurse acts in the guidelines about the side effects to the mastectomized patients who are in adjuvant chemotherapy treatment. The survey was conducted in UNACON (High Complexity Assistance Unit in Oncology) Institute of Nuclear Medicine and Endocrinology (IMNE / ONCOBEDA). The research sample consisted of 30 women who underwent mastectomy between 30 and 45 years. The research process was initiated by reading medical records and visits to the reffered Hospital for data collection. This was

applied to women. Quality of care and customer satisfaction were found in the guidelines provided by nurses, such as the most common side effects (alopecia, vomiting, nausea, cold sore, loss of appetite, diarrhea, depression, compromised peripheral venous access and anemia) and those that caused the most discomfort. We conclude that nurse has a key role in the guidelines provided to customers, clarifying doubts, facilitating their acceptance and their confrontation, thereby supplying all the needs of care provided during treatment.
\end{abstract} used to formulate a questionnaire subsequently

Keywords: Nursing; Mastectomy; Adjuvant Chemotherapy; Assistance of the nurse.

1 Universidade Estadual do Rio de Janeiro - UERJ - Rua São Francisco Xavier, 524 - Sala 1006 A - Maracanã, Rio de Janeiro - RJ, CEP: 20550-900, Brasil.

${ }^{2}$ Enfermagem do ISECENSA - Rua Salvador Correa, 139, Centro, Campos dos Goytacazes, RJ, CEP:28035-310, Brasil (*) e-mail: debora_tavaresfernandes@hotmail.com

Data de chegada: 14/11/2017 Aceito para publicação: 29/01/2018

Persp. online: biol. \& saúde, Campos dos Goytacazes, 26 (8), 19-30, 2018

seer.perspectivasonline.com.br 


\section{INTRODUÇÃO}

O câncer é uma doença crônica caracterizada por crescimento celular desordenado, o qual atinge órgãos e tecidos e se dissemina, através de metástases, pelo corpo. É considerada uma das doenças mais temidas no mundo inteiro e um problema de saúde pública, em nível mundial. Nesse contexto, a Organização Mundial da Saúde (OMS) estima que em 2030, pode-se esperar 27 milhões de casos incidentes de câncer, 17 milhões de mortes por câncer e 75 milhões de pessoas vivas com câncer (INCA 2011).

Segundo o INCA (2016), o câncer de mama se mantém como o segundo tipo de câncer com maior número de mortes nos países desenvolvidos, atrás apenas do câncer de pulmão, e maior causa de mortes nos países em desenvolvimento. É a maior causa de morte por câncer nas mulheres em todo o mundo, com aproximadamente 522 mil mortes estimadas para o ano de 2012, envolvendo em sua etiologia tanto fatores genéticos quanto ambientais ainda não totalmente conhecidos. No Brasil, é o tipo mais comum e representa a principal causa de mortalidade entre as mulheres.

A detecção precoce do nódulo mamário ainda é a maneira mais eficiente para a obtenção de tratamento e prognóstico satisfatórios. A prática do auto-exame é de extrema importância e fundamental para a detecção, sendo de fácil compreensão e acessível à mulher e dessa forma mostra-se muitas vezes, como uma forte arma contra a doença, impedindo a mutilação das mamas por meio da mastectomia e até mesmo a morte da paciente (BRASIL, 2012).

O impacto ocasionado pelo câncer na vida da pessoa é enorme, além da dor e do desconforto decorrentes da doença e seu tratamento, ocorrem mudanças de ordem psíquica, social e econômica (SILVA; HORTALE, 2006). Apesar dos avanços da medicina no tratamento do câncer e do aumento de informações veiculadas pela mídia, o câncer representa para alguns, a uma "sentença de morte", comumente associado à dor, sofrimento e degradação. O diagnóstico de câncer e todo o desenvolver da doença são vivenciados pela paciente e pela sua família como um momento de intensa angustia, sofrimento e ansiedade (VENÂNCIO, 2004).

Ao receber o diagnóstico da doença as pacientes passam por reflexões e questionamentos sobre a vida pregressa e futura à doença que afetarão diretamente seu modo de vida e seu comportamento em relação à própria saúde. A mama é associada ao símbolo de feminilidade, e a situação da doença associada à necessidade de uma mastectomia e posterior tratamento adjuvante que acarreta efeitos colaterais, acaba por afetar os mais diversos tipos de relacionamentos dessa paciente, fazendo com que a mulher sinta um incomodo real, que se estende aos familiares, fazendo-a viver uma sensação de tristeza, medo e negação (MELO et al., 2002).

Os processos cirúrgicos são os mais utilizados na terapêutica do câncer de mama, priorizando as demandas oncológicas essenciais para a manutenção da vida e, somente após, as questões estéticorreparadoras. Assim, há duas classificações: a cirurgia conservadora e a mastectomia. A cirurgia conservadora é o tratamento padrão para o câncer de mama em estádio inicial, e tem por objetivo a remoção do tumor com margens livres, preservando a maior parte da mama. A mastectomia, um procedimento que visa à retirada total da glândula mamária, com o objetivo de reduzir a incidência e melhorar a expectativa de vida de mulheres pertencentes a populações consideradas de alto risco, sendo quase sempre inevitável em fases adiantadas da doença (FRASSON; ZERWES, 2004).

Posteriormente a cirurgia, essas mulheres são submetidas às terapias sistemicas, como a quimioterapia, que apesar do impacto positivo aumentando a sobrevida das clientes, influenciam negativamente a qualidade de vida, devido aos efeitos adversos causados (NICOLUSSI; SAWADA, 2011).

Atualmente, a quimioterapia é, dentre as modalidades de tratamento, a que possui maior incidência de cura de muitos tumores incluindo os mais avançados, e a que mais aumenta a sobrevida dos portadores de câncer. Consiste em um tratamento sistêmico com utilização de agentes químicos, em combinação ou isolados, que tem por objetivo tratar os tumores malignos, se tornando uma das mais importantes maneiras de combate ao câncer, visando evitar a volta do tumor e o aparecimento em outros órgãos. Esta pode ser classificada quanto a sua finalidade em: curativa, adjuvante, neoadjuvante e paliativa, que serão empregadas

Persp. online: biol. \& saúde, Campos dos Goytacazes, 26 (8), 19-30, 2018 seer.perspectivasonline.com.br 
dependendo da extensão da doença, do tamanho do tumor e da condição física do paciente. A quimioterapia adjuvante é o tratamento que objetiva aumentar a chance de cura após determinado procedimento cirurgico, podendo ser ou não associado à radioterapia (BONASSA; GATO, 2012).

A associação do tratamento quimioterápico com o radioterapeutico e o cirúrgico potencializa a possibilidade de sobrevivência, uma vez que elimina ou previne as micrometástases. Porém, o ataque causado indiscriminadamente pela medicação às células de proliferação rápida, sendo estas normais ou cancerosas, resulta em efeitos tóxicos indesejáveis. Este fator torna o nível da qualidade de vida questionável, pelo fato dos efeitos colaterais serem agressivos na maioria das vezes, tanto no plano físico quanto no psicológico (BONASSA; GATO, 2012).

Segundo Gomes e Pisione (2012) e Camargo (1997), a quimioterapia está associada a efeitos colaterais responsáveis pela inabilidade física, como: anemia, fadiga, leucopenia, apatia, perda do apetite, alopecia, perda de peso, diarréia, hematomas, mucosite, náuseas e vômitos. Além de mudanças na imagem corporal, alterações emocionais e do cotidiano. Mesmo com todos estes efeitos é importante a continuidade para o sucesso do tratamento.

Nesse sentido, a mulher mastectomizada, quando em tratamento quimioterápico, tende a ficar mais sensível e vulnerável aos estímulos que o mesmo provoca, muitas vezes, interferindo na promoção da resposta eficaz ao tratamento. A mastectomia costuma causar impacto, abalando a auto-estima desta mulher. Quando associada à quimioterapia, esse é agravado ainda mais em decorrência dos efeitos colaterais, especialmente à queda de cabelo, podendo evidenciar respostas ineficazes que se refletem em medo, depressão, angústia, nervosísmo e tristeza (MISTURA; CARVALHO; SANTOS, 2011).

Paciente e familiares devem estar plenamente orientados sobre as toxicicidades relacionadas ao tratamento, através de informações verbais e escritas assimililadas, transmitidas com respeito e empatia, sempre reforçando os beneficios das drogas e as alternativas para o manejo das reações indesejáveis (BONASSA; GATO, 2012).

Os profissionais enfermeiros reconhecem a importância dos diversos tratamentos do câncer, porém a assistência não deve ser apenas o manejo dos efeitos colaterais, mas se estender ao ambiente construído ao seu redor. Deve compreender como a doença e o tratamento afetam a paciente e como ela as interpreta (ANJOS; ZAGO, 2006). Nesse contexto, a enfermeira aprende com o doente a sua história, perspectiva pessoal, enquanto o doente recebe da enfermeira elementos de natureza cognitiva e outros que lhe facilitam o lidar com a situação (LOPES, 2005).

Este estudo tem como objetivo enfatizar a importância na qualidade das orientações prestadas pelo profissional enfermeiro a essas mulheres, acerca dos efeitos colaterais que poderão ocorrer durante o tratamento quimioterápico adjuvante, fornecendo informações que poderão ajuda-las a lidar com o mesmo.

\section{METODOLOGIA}

Trata-se de um estudo qualitativo de caráter exploratório.

A pesquisa foi realizada na UNACON (Unidade Assistência de Alta Complexidade em Oncologia) do Instituto de Medicina Nuclear e Endocrinologia (IMNE/ ONCOBEDA), com característica: pública e conveniada, sendo este um Hospital de referência regional no tratamento de câncer, localizado no Município de Campos dos Goytacazes/ RJ.

A amostra da pesquisa foi formada por 30 mulheres mastectomizadas entre 30 e 45 anos. Foram utilizados como critério de exclusão: mulheres portadoras de neoplasia benigna, fora da faixa etária estipulada, mastectomizadas que não estejam em tratamento, sem confirmação diagnóstica e iniciado o tratamento a menos de 48 horas. Foi utilizado como critério de inclusão as mulheres que se enquadram na faixa etária e em tratamento quimioterápico adjuvante, garantindo assim, rigor metodológico à pesquisa.

Esta pesquisa foi submetida para apreciação do Comitê de Ética em Pesquisa com seres humanos do

Persp. online: biol. \& saúde, Campos dos Goytacazes, 26 (8), 19-30, 2018

seer.perspectivasonline.com.br 
ISECENSA (CEPISECENSA), sendo aprovada pelo protocolo ${ }^{\circ}$. 0065.0.413.000-11.

O processo de pesquisa foi realizado pelas graduandas do curso de enfermagem do Isecensa, não integrantes da equipe de enfermagem do hospital, responsáveis pela mesma. A presente pesquisa foi iniciada através da leitura dos prontuários de pacientes em tratamento no UNACON, a fim de identificar as mulheres que se enquadram nos critérios de inclusão acima estabelecidos, por meio da coleta de dados.

Após a definição da amostra foram realizadas visitas ao referido Hospital, a fim de acompanhar a rotina da assistência do enfermeiro prestada a essas mulheres, visando a elaboração do questionário através deste contato. Neste período, foi estabelecido o primeiro contato com as mulheres participantes da pesquisa, onde ocorreu a aplicação do Termo de Consentimento Livre e Esclarecido (TCLE). Na segunda etapa da pesquisa, a coleta de dados foi utilizada para formulação de um questionário, posteriormente aplicado às mulheres, que visou analisar a presença e a qualidade das orientações prestadas pelo profissional enfermeiro às clientes.

Foi assegurado o sigilo da identidade dos participantes, bem como das informações fornecidas pelos mesmos, garantido pelo Código de Ética de Enfermagem, Capítulo II do Sigilo Profissional. Posteriormente, os dados foram analisados criticamente, utilizando a estatistica descritiva com variável qualitativa e, receberam tratamento através de gráficos, nos quais poderá se demonstrar a amplitude da assistência do profissional enfermeiro nas orientações acerca dos efeitos colaterais recorrentes do tratamento, e como as clientes reagem a eles.

Os riscos para as envolvidas na pesquisa são nulos, e os benefícios estão centrados na formulação de um plano de cuidados, se preciso, a partir das respostas que estão no questionário, onde foi analisado o nível da assistência de enfermagem prestada através das orientações e a satisfação das mulheres em relação ao atendimento prestado pelo profissional enfermeiro, a fim de proporcionar uma melhor assistência e por conseqüência uma melhor qualidade de vida à essas mulheres.

Os questionários aplicados e os dados coletados serão armazenados em arquivos impressos para análise crítica qualitativa das informações obtidas.

\section{RESULTADOS}

O referido Hospital, por se tratar de um local de referência, proporciona tratamentos com medicamentos que fazem parte de pesquisas clínicas. Torna-se importante que o enfermeiro oncológico tenha conhecimento dos protocolos, das reações adversas, toxicidades, doses, tempo de infusão para que assim, possa realizar um monitoramento adequado dos pacientes.

As clientes que chegam à unidade para iniciarem o tratamento quimioterápico, são recepcionadas pelo profissional enfermeiro, que mostrou além de empatia, preparo científico para prestar todas as informações necessárias ao esclarecimento das dúvidas existentes.

Ao se deparar com o diagnóstico da doença e a necessidade da realização de uma mastectomia e posterior tratamento adjuvante que traria inúmeros efeitos colaterais, todas relataram que até ocorrer à aceitação do câncer houve uma série de mudanças e um forte impacto em diversos segmentos da sua vida. Ficou evidente que em muitos momentos a severidade dos efeitos colaterais trouxe à tona uma enorme sensação de impotência, que em muitos momentos se sobrepunha a vontade de viver.

A análise dos dados coletados através dos questionários mostrou a eficiência na assistência de enfermagem prestada às clientes no inicio e ao longo do tratamento. As orientações acerca dos efeitos colaterais e como deve ser o manejo dos mesmos são prestadas com clareza pelo profissional enfermeiro no momento em que a cliente inicia o tratamento, levando ao esclarecimento das dúvidas encontradas a respeito desses efeitos. Orientações essas, como: o tipo de alimentação adequada, quais efeitos poderão ocorrer e o tempo estimado para a manifestação dos mesmos, quais atitudes devem ser tomadas durante o aparecimento de cada efeito colateral, e como proceder durante uma situação que não esteja prevista nos padrões "normais" do tratamento, esta deve ser comunicada imediatamente ao enfermeiro. Essa comunicação geralmente é realizada

Persp. online: biol. \& saúde, Campos dos Goytacazes, 26 (8), 19-30, 2018

seer.perspectivasonline.com.br 
através de ligação telefônica para o aparelho móvel do profissional, o número é deixado pelo mesmo à disposição das clientes e familiares para que possam informar o ocorrido e as orientações possam ser passadas. Quando outras dúvidas acerca dos efeitos vão surgindo durante o tratamento, essas podem ser esclarecidas tanto pelo telefone móvel, como através da consulta de enfermagem, sendo dentre estes, o aparelho móvel o meio mais utilizado.

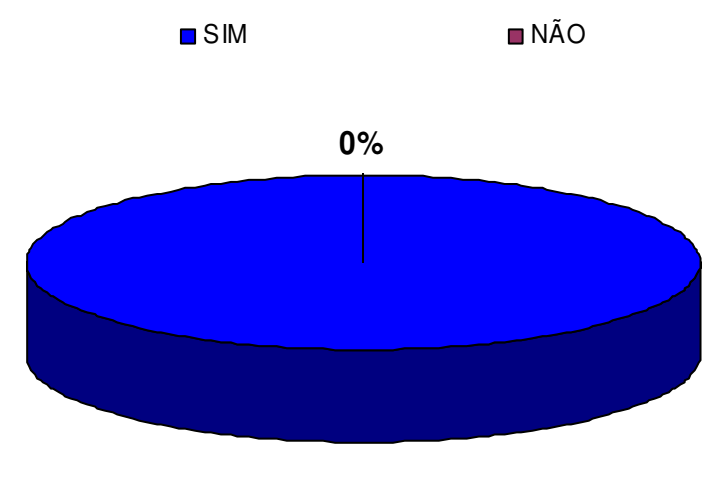

$100 \%$

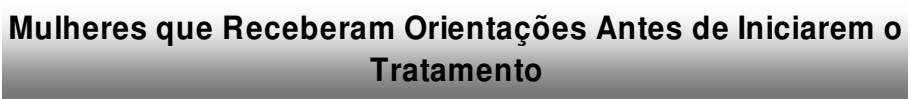

Figura 1: Percentual de mulheres que foram orientadas pelo enfermeiro sobre os efeitos colaterais que podem ocorrer ao iniciar o tratamento quimioterápico. As orientações foram realizadas através da consulta de enfermagem.
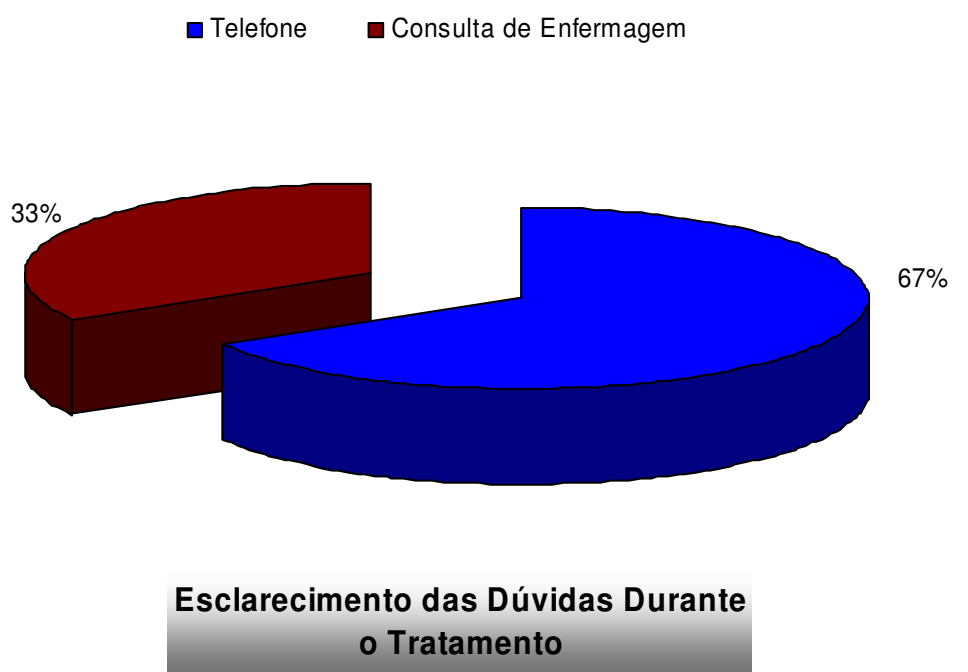

Figura 2: Estratégias de comunicação utilizadas entre profissional enfermeiro e pacientes para esclarecimentos de dúvidas no decorrer do tratamento.

Pode-se constatar dentre os efeitos colaterais mais comuns que as clientes em tratamento quimioterápico adjuvante podem apresentar, quais foram os mais freqüentes entre elas e os que causaram maior incômodo. Entre os mais freqüentes, estão: alopecia, vômito, náuseas, afta, perda de apetite, diarréia, depressão, acesso venoso periférico comprometido (AVPC) e anemia, dentre estes, a alopecia, vômito, afta, depressão e náuseas foram os efeitos que mais incomodaram. As clientes relataram que as orientações prestadas pelo enfermeiro sobre os efeitos colaterais antes e durante o tratamento foram e continuam sendo de 
fundamental importância para que elas possam estar preparadas física e emocionalmente para enfrentarem com consciência mais essa etapa do processo.

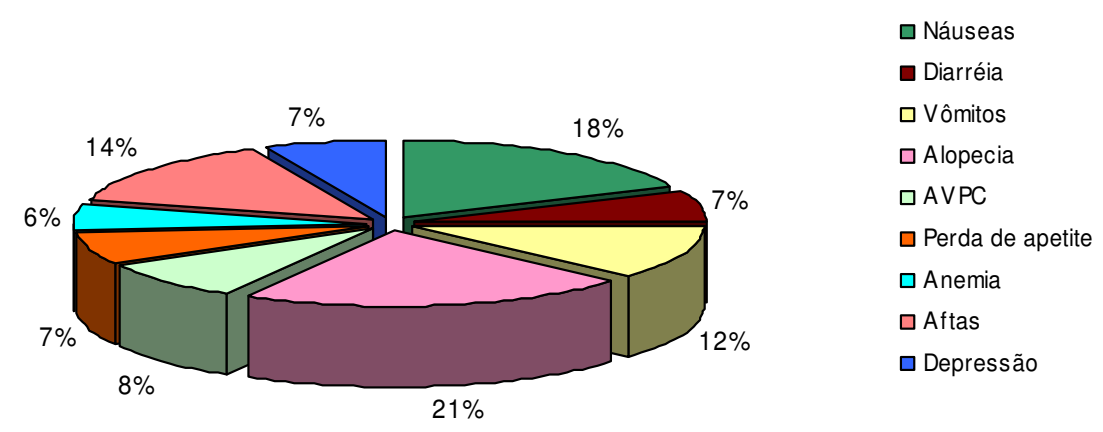

Efeitos colaterais mais frequentes

Figura 3: Porcentagem dos efeitos colaterais mais apresentados entre as 30 mulheres envolvidas na pesquisa. A sigla AVPC representa Acesso Venoso Periférico Comprometido.

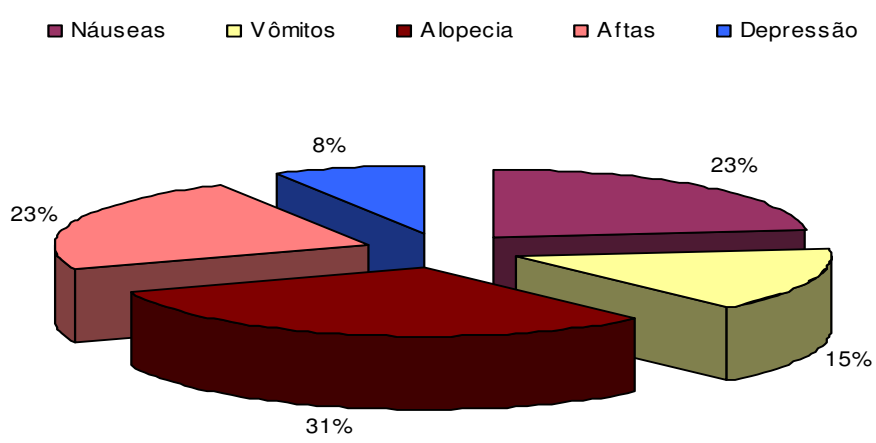

Efeitos coletarais de maior incômodo

Figura 4: Porcentagem dos efeitos colaterais que mais incomodaram as mulheres envolvidas na pesquisa. Estes foram escolhidos entre os efeitos considerados mais freqüentes.

Mesmo com as orientações prestadas a fim de "preparar" estas mulheres para enfrentar o tratamento, diante dos efeitos colaterais (agressivos na maioria das vezes), constatou-se uma grande debilidade emocional entre a maioria, demonstrada através do sentimento de impotência, a não aceitação da doença, o questionamento, "convicção" da perda da feminilidade (devido a retirada da mama e da alopecia), e o medo da morte, levando-as a necessidade de um apoio emocional por parte de outros indivíduos além do profissional enfermeiro e de todos os outros profissionais de saúde envolvidos no tratamento (médico, fisioterapeuta, psicólogo, nutricionista, etc.). Estes indivíduos foram classificados pelas mulheres, como sendo de muita importância no decorrer do processo de quimioterapia, sendo eles: a família, os amigos e a equipe de funcionários do ONCOBEDA (classificados no questionário como outros). Todos eles de acordo com as clientes têm importância no papel que exercem dentro deste processo de tratamento quimioterápico adjuvante.

Persp. online: biol. \& saúde, Campos dos Goytacazes, 26 (8), 19-30, 2018

seer.perspectivasonline.com.br 


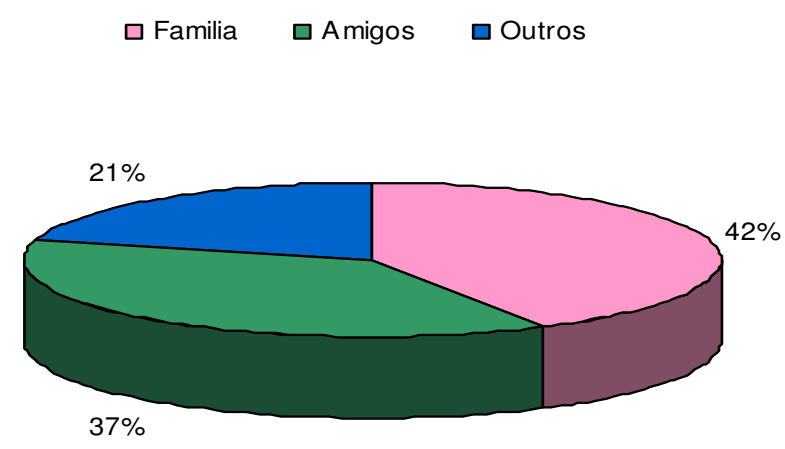

Pessoas consideradas importantes

Figura 5: Porcentagem dos indivíduos classificados pelas mulheres como importantes no decorrer do tratamento, além dos profissionais de saúde envolvidos no mesmo. Os individuos classificados como OUTROS representam os funcionarios do ONCOBEDA.

As mulheres relataram satisfação na assistência prestada pelo enfermeiro. Todas as pacientes avaliaram como boa a excelente a atuação do enfermeiro a respeito do tipo de tratamento, duração e também acerca dos efeitos colaterais que poderiam vir a ocorrer a curto, médio e longo prazo, ressaltando a todo momento o quanto acham importante a humanização do atendimento aliado ao entrosamento da equipe multiprofissional. Ressaltando que para a assistência ser prestada com eficiência, torna-se necessário que o enfermeiro possua conhecimento científico acerca de todos os processos da doença e do tratamento, para que assim, haja uma resolutividade na suas ações. (Fígura 6)

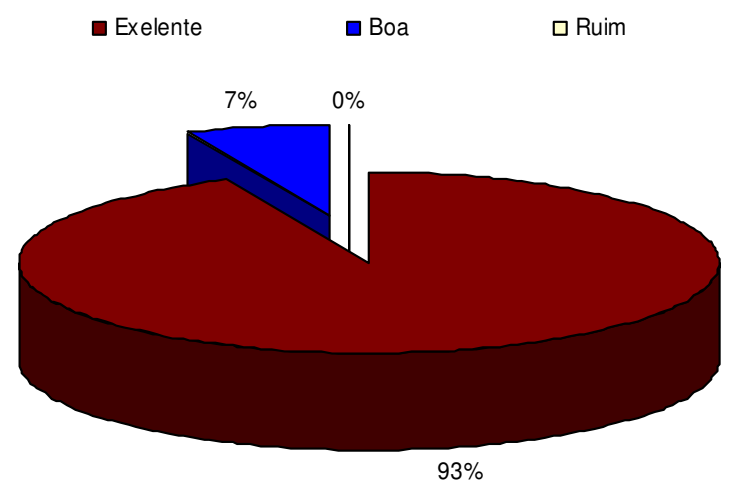

\section{Classificação da Assistência}

Figura 6: Classificação realizada pelas mulheres envolvidas na pesquisa, sobre o nível da assistência e orientações prestadas pela equipe de enfermagem.

\section{DISCUSSÃO}

O profissional enfermeiro, por ficar mais perto e mais tempo com os clientes e familiares, encontra-se em uma posição de destaque para melhor cuidar dos mesmos. A especialidade de enfermagem oncológica, nas ultimas décadas, vem ganhando amplitude e profundidade. Todavia, em vista da complexidade do paciente oncológico, pelos aspectos físicos e psicossociais abordados, o enfermeiro é o único qualificado para lidar com todos estes aspectos. Há de se considerar que suas funções refletem sua formação profissional, e suas atuações devem ser adaptadas ao tipo da população atendida, conforme a sua experiência profissional (BONASSA; GATO, 2012).

É válido salientar a importância da assistência de enfermagem individualizada, na medida que se verifica, na população atendida até o momento, a alta taxa de adesão ao tratamento proposto, por meio da

Persp. online: biol. \& saúde, Campos dos Goytacazes, 26 (8), 19-30, 2018

seer.perspectivasonline.com.br 
consulta de enfermagem e ausência de casos de abandono ao atendimento ambulatorial. É notória a importância da assistência de enfermagem individualizada na melhoria na qualidade de vida dos pacientes, na diminuição dos efeitos colaterais e do número de internações decorrentes de complicações advindas da quimioterapia, além do bem-estar referidos por pacientes e familiares atendidos (ANJOS et al., 2011).

Durante a primeira consulta de enfermagem, onde ocorrem as orientações sobre o decorrer do tratamento e os efeitos colaterais ocasionados pelo mesmo, as mulheres apresentam sinais evidentes de ansiedade e medo, sentimentos que se referem ao desconhecido, ao novo, aliados o estigma do câncer (GARGIULO et al, 2007).

O tratamento quimioterápico pode ocasionar interferências negativas em todo o organismo, devido a sua natureza sistêmica, o que contribui para a diminuição da qualidade de vida relacionada à saúde. Os efeitos colaterais geram desequilíbrios, que dificultam o cumprimento do regime terapêutico, possibilitando a diminuição da efetividade do tratamento, gerando ainda ansiedade e maior desgaste físico e psicológico nas pacientes e familiares. Por isso acredita-se que o paciente que recebe as orientações tem maiores chances de diminuição dos efeitos colaterais e reações adversas, assim sendo, terá maiores possibilidades de cumprir todas as etapas e ciclos do tratamento estabelecido (BORGES; ANJOS, 2010).

Possuir domínio científico é indidpensável para o profissional enfermeiro oncológico, tornando necessário que o mesmo tenha conhecimento dos protocolos, das reações adversas, toxicidade, doses, tempo de infusão, para que assim possa realizar o monitoramento e orientações adequadas às clientes. (FORONES, 2005).

Deste modo, o conhecimento sobre os efeitos adversos dos quimioterápicos é fundamental para o planejamento do cuidado de enfermagem. Estudos têm enfatizado a importância das orientações no melhor enfrentamento destes efeitos colaterais e das reações adversas possíveis, trazendo subsídios adequados para a prevenção e manejo. Cabe ao enfermeiro indicar e fornecer orientações relativas as medidas preventivas, identificar precocemente os efeitos colaterais do taratmento a fim de minimizá-los, orientar e acompanhar a paciente e respectiva família. (FRIGATO; HOGA, 2003).

Diante de experiências vividas e frente a trabalhos de pesquisa já desenvolvidos com este foco, acredita-se que a consulta de enfermagem, e a prática de ações que envolvam orientações específicas e individualizadas sejam importantes para maior adesão dos pacientes ao tratamento e para o enfrentamento das reações adversas, tendo em vista que os efeitos colaterais mais comuns, decorrentes da ação de agentes quimioterápicos estão relacionados ao estado nutricional de cada mulher. Seus efeitos dependem do medicamento, da dose e de utilização de outras formas de tratamento concomitantemente (COTRIM et al., 2003).

É de responsabilidade do profissional enfermeiro prestar informações sobre a doença, os cuidados após quimioterapia e orientações sobre efeitos colaterais da medicação. O Código de Ética dos Profissionais de enfermagem, em seu artigo 26, prevê o dever de prestar adequadas informações ao paciente e à sua família a respeito da assistência de enfermagem, possíveis riscos e consequiências que possam ocorrer. (COFEN, 2000).

Ao receber o diagnóstico de câncer da mama, a mulher precisará do apoio familiar, e é necessário que a família não se esqueça de suas próprias necessidades, sendo solidária, não permitindo que a paciente se deixe vencer pela doença. $\mathrm{O}$ afeto familiar permite à mulher manter uma certa estabilidade para lutar contra $\mathrm{a}$ doença. Sendo assim, consegue suprir suas carências emocionais, alcança uma melhor aceitação e orientação comportamental. Neste sentido, nenhuma pessoa vive no mais completo isolamento, sem ser influenciada pelo meio em que vive e pelas pessoas que a rodeiam (BERVIAN; PERLINI, 2006).

A doença associada à necessidade da mastectomia e, posteriormente ao processo quimioterápico/radioterápico resulta numa série de alterações de ordem emocional, física e social à vida dessas mulheres, além de acarretar efeitos colaterais que afetam os mais diversos tipos de relacionamentos dessa cliente, causando-a um incômodo real que se estende aos familiares. Diante da grande incidência do câncer de mama no Brasil e a desestruturação que o diagnóstico traz à vida dessa mulher e sua família, torna-

Persp. online: biol. \& saúde, Campos dos Goytacazes, 26 (8), 19-30, 2018 seer.perspectivasonline.com.br 
se importante que o enfermeiro desenvolva estratégias de atenção para ambos (MELO; SILVA; FERNANDES, 2005).

\section{CONCLUSÕES}

A partir do presente estudo, pode-se perceber a importância das orientações do enfermeiro, através da consulta de enfermagem, para que as mulheres mastectomizadas em tratamento quimioterápico adjuvante tenham conhecimento prévio sobre o tratamento e um papel ativo no manejo dos efeitos colaterais, realizando o auto-cuidado, impactando de maneira positiva na qualidade de vida. Contudo, é de extrema importância que as orientações sejam embasadas em aspectos científicos, sendo o enfermeiro responsável por elaborar estratégias que tenham como objetivo a adesão às orientações para que possam resultar em maior eficácia.

Diante dos resultados apresentados e das análises realizadas, observa-se que as orientações prestadas na consulta de enfermagem tornam as mulheres preparadas para enfrentar da melhor maneira os efeitos colaterais decorrentes do tratamento. Pode-se perceber o quanto as ações dos profissionais de saúde são importantes diante das necessidades apresentadas pelas pacientes. Diante dos mais diversos sentimentos e questionamentos que são trazidos à tona pela situação vivida por essas mulheres, o enfermeiro tem papel primordial nas orientações prestadas às clientes: esclarecendo dúvidas, facilitando a aceitação e suprindo necessidades de cuidado.

Frente a esse quadro, torna-se evidente a necessidade do profissional enfermeiro estar bem qualificado para assistir cada mulher em sua integralidade, fornecendo orientações necessárias acerca dos efeitos colaterais e do tratamento, através da consulta de enfermagem, prestando assistência com eficiência e qualidade.

Conforme relatado pelas pacientes, uma equipe qualificada e dedicada transforma o viver com câncer numa luta onde é possível enxergar as mais diversas possibilidades de vitória.

\section{REFERÊNCIAS}

ANJOS, A.C.Y; MAGNABOSCO, P; BORGES, B.O; CAMPOS, C.S: Sistematização da assistência de enfermagem ao paciente em tratamento quimioterápico antineoplasico: Relato de experiencia. Em Extensão, v.10 n.1 107-112.p.: Uberlândia, jan/jun. 2011. Disponível em: $<$ http://www.revistadeextencao.proex.ufu.br>

ANJOS, A.C.Y \& ZAGO, M.M.F. A experiência da terapêutica quimioterápica oncológica na visão do paciente. Revista Latino-americana de Enfermagem. janeiro-fevereiro; 14(1):33-40. 2006. Disponível em: $<$ http://www.scielo.br>

BERVIAN, P.I \& PERLINE, N.M.O.G. A família (con)vivendo com a mulher/mãe após a mastectomia. Revista Brasileira de Cancerologia 2006; 52(2): 121/128. Disponível: <http://www.L.inca.gov.br>

BONASSA, E.M.A \& GATO, M.I.R. Terapêutica Oncológica para Enfermeiros e Farmacêuticos. 4. ed. Atheneu: São Paulo, Rio de Janeiro, Belo Horizonte, 2012.

BRASIL, MS. Ações de Prevenção Primária e Secundária no Controle do Câncer de Mama. Rio de Janeiro: INCA, 2012.

BRASIL, MS \& INSTITUTO NACIONAL DO CÂNCER. Estimativa 2016: incidência de câncer no Brasil / Instituto Nacional de Câncer José Alencar Gomes da Silva - Rio de Janeiro: INCA, 2015. Disponível em: <http://www.inca.gov.br/estimativa/2016/estimativa-2016-v11.pdf>

BORGES, D.O \& ANJOS, A.C.Y. Sistematização da Assistência de Enfermagem ao Paciente em Tratamento Quimioterápico: Adesão às orientações e a efetividade na diminuição de efeitos colaterais e 
atrasos no tratamento. Faculdade de Medicina da Universidade Federal de Uberlândia. Minas Gerais, 2010. Disponivel em: <http://www.seer.ufu.br>

CAMARGO, T.C. O ex-sistir feminino num rosto sem moldura: uma análise compreensiva. Escola de Enfermagem Ana Nery, UFRJ. Nov.p.146. Rio de Jneiro, RJ, 1997. Disponível em: htt://bvsms.saude.gov.br

COFEN, CONSELHO FEDERAL DE ENFERMAGEM. Código de Etica dos profissionais de Enfermagem. Capitulo V os deveres, artigo 26. Resolução COFEN - 240/2000, revogada pela Resolução COFEN 311/2007. Disponível em : <http://novo.portalcofen.gov.br>

COTRIN, T.H; IKEMORI, E.H.A.; OLIVEIRA, T.; SERRALHEIRO, I.F.D.; SHIBUYA; E \& TRINTIN, L.A. Acompanhamento nutricional de pacientes em radioterapia e quimioterapia. Nutrição em oncologia. $1^{\text {a }}$ edição. SP: Lemar; 2003.p.205-18.

FORONES, N.M. Manual de oncologia. SP: Manole, 2005.

FRASSON, A.L \& ZERWES F.P. Câncer de mama. Psicooncologia e interdisciplinaridade: uma experiência na educação à distância. Edipucrs: Porto Alegre. p. 95-108. 2004

FRIGATO, S. \& HOGA, L.A.K. Assistencia a Mulher com Câncer de colo de útero: O papel da enfermagem. 2003. Disponível em: <http://www.inca.gov.br/>

GARGIOLO C.A; MELO M.C.S.C; TALIMENA, A.M.O; BARRA, V.M.S \& SOUZA, E.O. Vivenciando o cotidiano na percepção de enfermeiras oncológicas. Texto \& Contexto- Enf v.16 N.4 Florianópolis out/dez 2007. Disponível em : <http://scielo.br>

GOMES; J.S \& PISONI A.C. Dificuldades vivenciadas por mulher em tratamento para o câncer de mama. Universeidade Regional do nordeste do Estado do Rio Grande do Sul - UNIJUI: Ijui, RS. 2012. Disponível em: http://www.esenfc.pt

GUTIÉRREZ, M.G.R. et.al. Natureza e Classificação das internações de enfermagem em ambulatório de quimioterapia de adultos. 200. Disponível em: <http://www.revistasusp.sibi.usp.br/>

INSTITUTO NACIONAL DO CÂNCER. Ações de enfermagem para o controle do câncer: uma proposta de integração ensino-serviço/Instituto Nacional de Câncer. 3 ed. Ver. Atual Ampl. Rio de Janeiro: INCA, 2008.

LOPES, M.J. Os clientes e os enfermeiros: construção de uma relação. Rev.esc.enferm USP. V.39 n.2: São Paulo, 2005. Disponível em: <http://www.scielo.com>

MELO, E.M; ARAÚJO, T.L; OLIVEIRA, T.C \& ALMEIDA, D.T. Mulher mastectomizada em Tratamento Quimioterápico: um estudo dos comportamentos na perspectiva do modelo adaptativo dos Roy's. Revista Brasileira de Cancerologia. 48(1): 21-28. 2002. Disponível em: <http://www.inca.gov.br>

MELO, E.M: SILVA, R.M \& FERNANDES, A.F.C. Orelacionamento familiar após a mastectomia: um enfoque no modelo de interdependencia de Roy. Revista Brasileira de cancerologia, 2005; 51(3): 2019/225. Disponível em: <http://www.inca.gov.br>

MISTURA, C. CARVALHO, M.F.A.A. \& SANTOS, V.E.P. Mulheres mastectomizadas: Vivências frente ao câncer de mama. Rev. Enferm. UFSM. Set/Dez;1(3):351-359. 2011. Disponível em: $<$ http://cascavel.ufms.br>

NICOLUSSI, A.C \& SAWADA, N.O. Qualidade de vida de pacientes com câncer de mama em terapia adjuvante. Rev Gaúcha Enferm. dez; 32(4):759-66. Porto Alegre, 2011. Disponível em: $<$ http://www.scielo.br> 
SILVA, R.C.F \& HORTALE, V. Cuidados paliativos oncológicos: elementos para o debate de diretrizes nesta área. Cad de Saúde Pública. 22(10). 2006. Disponível em: <http://www.scielo.br>

VENÂNCIO, J.L. Importancia da atuação do psicólogo no tratamento de mulheres com câncer de mama. Revista brasileira de cancerologia. Vol.50. $n^{\circ} 1.55 / 63$ p.2004. Disponível em: <http://www.inca.gov.br> 